\title{
Proof of Concept for an Ultrasensitive Technique to Detect and Localize Sources of Elastic Nonlinearity Using Phononic Crystals
}

\author{
M. Miniaci, ${ }^{1}$ A. S. Gliozzi, ${ }^{2, *}$ B. Morvan, ${ }^{1}$ A. Krushynska, ${ }^{3}$ F. Bosia, ${ }^{3}$ M. Scalerandi, ${ }^{2}$ and N. M. Pugno ${ }^{4,5,6}$ \\ ${ }^{1}$ University of Le Havre, Laboratoire Ondes et Milieux Complexes, UMR CNRS 6294, 75 Rue Bellot, 76600 Le Havre, France \\ ${ }^{2}$ Department of Applied Science and Technology, Politecnico di Torino, Corso Duca degli Abruzzi 24, 10129 Torino, Italy \\ ${ }^{3}$ Department of Physics and Nanostructured Interfarces and Surfaces Centre, University of Torino, \\ Via Pietro Giuria 1, 10125 Torino, Italy \\ ${ }^{4}$ Laboratory of Bio-Inspired and Graphene Nanomechanics, Department of Civil, Environmental and Mechanical Engineering, \\ University of Trento, Via Mesiano 77, 38123 Trento, Italy \\ ${ }^{5}$ School of Engineering and Materials Science, Queen Mary University of London, Mile End Road, London E1 4NS, United Kingdom \\ ${ }^{6}$ Ket Lab, Edoardo Amaldi Foudation, Italian Space Agency, Via del Politecnico snc, 00133 Rome, Italy
}

(Received 23 December 2016; published 26 May 2017)

\begin{abstract}
The appearance of nonlinear effects in elastic wave propagation is one of the most reliable and sensitive indicators of the onset of material damage. However, these effects are usually very small and can be detected only using cumbersome digital signal processing techniques. Here, we propose and experimentally validate an alternative approach, using the filtering and focusing properties of phononic crystals to naturally select and reflect the higher harmonics generated by nonlinear effects, enabling the realization of time-reversal procedures for nonlinear elastic source detection. The proposed device demonstrates its potential as an efficient, compact, portable, passive apparatus for nonlinear elastic wave sensing and damage detection.
\end{abstract}

DOI: 10.1103/PhysRevLett.118.214301

In recent years, phononic crystals (PCs) have attracted great attention due to their unconventional dynamic behavior, with effects such as negative refraction [1], frequency band gaps [2,3], wave filtering or focusing [4-6], acoustic cloaking [7-9], subwavelength sensing [10,11], etc. Their periodic structure, rather than single material constituents, is responsible for their behavior, which exploits Bragg scattering $[12,13]$. Their attractive properties to act as stopband filters [12] or to concentrate energy in selected frequency ranges [14] makes them potentially interesting for nonlinear elastic source detection and to reveal the presence of defects, e.g. cracks, in a sample. This is because, in general, a nonlinear response is generated at the defect location and several possible features may appear, including the generation of higher order harmonics [15-17] or subharmonics [18,19], the nonlinear dependence of the elastic modulus and of attenuation coefficients on strain [20-22], and, as a consequence, the shift of the resonance frequency with increasing excitation amplitude $[23,24]$ and the failure of the superposition principle $[25,26]$. All of these possible signatures can be used to detect and monitor the presence and evolution of damage, exploiting the greater sensitivity of nonlinear detection techniques compared to conventional linear ones [27].

In the past years, nonlinear imaging techniques such as $b$ scan, $c$ scan, and tomography [28] have attracted much interest. A particularly robust and efficient approach is the combination of time reversal (TR) and nonlinear elastic wave spectroscopy (NEWS). This technique (TR-NEWS) exploits space-time focusing of the wave field achieved in TR [29] and applies it to a defect acting as a source of nonlinear elastic waves [30-33]. The scattered signal is recorded, the frequency generated by the primary source is filtered out using a bandpass filter, and the resulting signal is time reversed and reinjected by the receiver: due to the $(t \rightarrow-t)$ symmetry, the wave field back propagates to its original (nonlinear) source, focusing energy at the defect location at a specific time. Many studies have proved the efficiency and robustness of TR-NEWS in various configurations, for different types of nonlinear sources [34-36] and in assorted experimental conditions [31]. However, TR-NEWS relies-as do most of the techniques for both the detection and the location of damage-on extensive signal manipulation (normally, digital filtering), which might be critical in the case of short signals and/or when continuous signal acquisition is required (such as in acoustic emissions). Furthermore, the nonlinear components of the wave field are often very small, if not submerged by the noise level, making it difficult to detect and estimate them. The concept adopted in this Letter overcomes these limitations, combining TR-NEWS and phononic crystals in order to introduce a technique capable of filtering out and concentrating energy in target frequency ranges. We experimentally demonstrate the feasibility and the efficiency of this technique, providing the proof of concept for an ultrasensitive phononic crystal device to detect and localize nonlinear elastic sources such as cracks or delaminations.

A schematic representation of the experimental setup is given in Fig. 1. The sample is a pristine $300 \times 300 \times 3 \mathrm{~mm}^{3}$ aluminum plate (the density $\rho=2700 \mathrm{~kg} / \mathrm{m}^{3}$, the Young's modulus $E=70 \mathrm{GPa}$, and the Poisson's ratio $\nu=0.33$ ), 


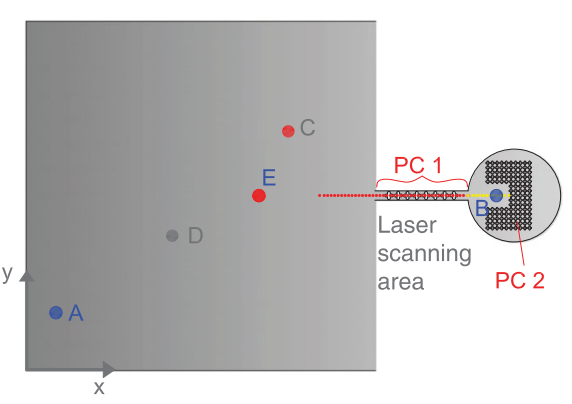

(a)

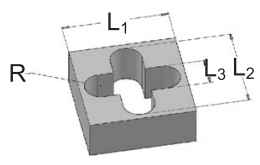

(b)

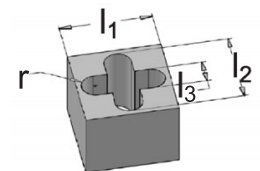

(c)
FIG. 1. (a) Schematic representation of the specimen composed of an aluminum plate connected to a filtering phononic crystal region (PC1) and a chaotic cavity [37] with an additional focusing phononic crystal-based structure (PC2). Three-dimensional view of the unit cells for (b) the PC1 region and (c) the PC2 region.

attached to which is a device, consisting of two phononic crystal regions (referred to as PC1 and PC2, respectively). PC1 consists of a 1D array of eight crosslike cavities cut in a narrow rectangular waveguide and PC2 of a $C$-shaped array of smaller unit cells. The cavities are fabricated using waterjet cutting, with different lattice parameters depending on the filtering [Fig. 1(b)] or reflecting [Fig. 1(c)] function that they are designed for. Specifically, the lattice parameters are $L_{1}=8 \mathrm{~mm}, L_{2}=0.9 L_{1}$, and $L_{3}=2 R=0.3 L_{1}$ for PC1 and $l_{1}=4 \mathrm{~mm}, l_{2}=0.9 l_{1}$, and $l_{3}=2 r=0.3 l_{1}$ for $\mathrm{PC} 2$, respectively. A crosslike geometry is chosen because it allows large band gap (BG) nucleation [38].

In order to investigate the $\mathrm{BG}$ structure of the $\mathrm{PC} 1$ region, its transmission spectrum is first investigated in a pitch-catch experiment [38] according to the schematic representation of Fig. 1(a) (see Ref. [39] for details). An ultrasonic pulse with a frequency content between 50 and $450 \mathrm{kHz}$ is launched by a transducer attached to the top

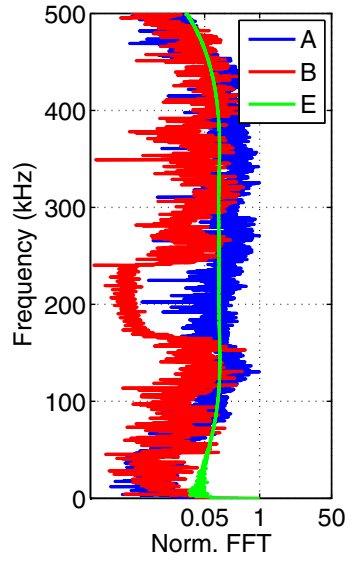

(a)

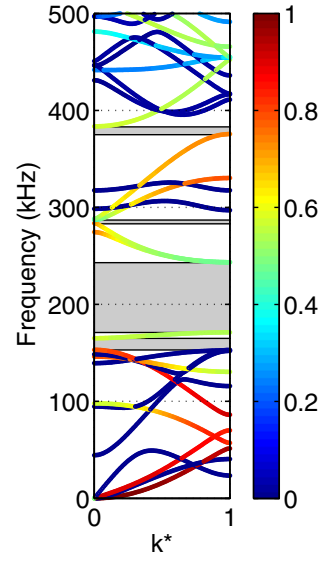

(b)
FIG. 2. (a) Experimental normalized transmission frequency spectrum and (b) the corresponding numerically predicted dispersion band structure for the $\mathrm{PC} 1$ region. Mode polarization is indicated by color, ranging from pure in plane (blue) to pure out of plane (red). surface of the plate [point $E$ in Fig. 1(a)] and received at points $A$ and $B$ using 5-mm-diameter piezoelectric disk sensors. Figure 2(a) shows the fast Fourier transform (FFT) of the input signal in $E$ (the green line) and those recorded in $A$ and $B$ (the blue and red lines, respectively). A large BG appears between 172 and $244 \mathrm{kHz}$, highlighted by a considerable frequency drop at the corresponding frequencies (up to $100 \mathrm{~dB}$ ). Three smaller BGs are visible around 153,285 , and $380 \mathrm{kHz}$. On the contrary, the spectral content of the signal recorded in the plate [the blue line in Fig. 2(a)], which is not subject to any filtering, shows the same frequency content as the excitation. These results are in agreement with numerically computed dispersion diagrams using Bloch-Floquet theory [41] in full 3D FEM simulations [Fig. 2(b)]. Here, the band structure is shown in terms of the reduced wave vector $k^{*}=\left[k_{x} L_{1} / \pi ; k_{y} L_{1} / \pi\right]$, varying along the first irreducible Brillouin zone boundary $\Gamma-X$, with colors indicating mode polarization [39]. The BGs are highlighted in grey.

Further information on the dynamical properties of the PC1 region is obtained by injecting a short pulse at point $E$ and using a scanning laser Doppler vibrometer (SLDV) to measure the out-of-plane component of the velocity at the surface along the dotted path highlighted in Fig. 1(a)

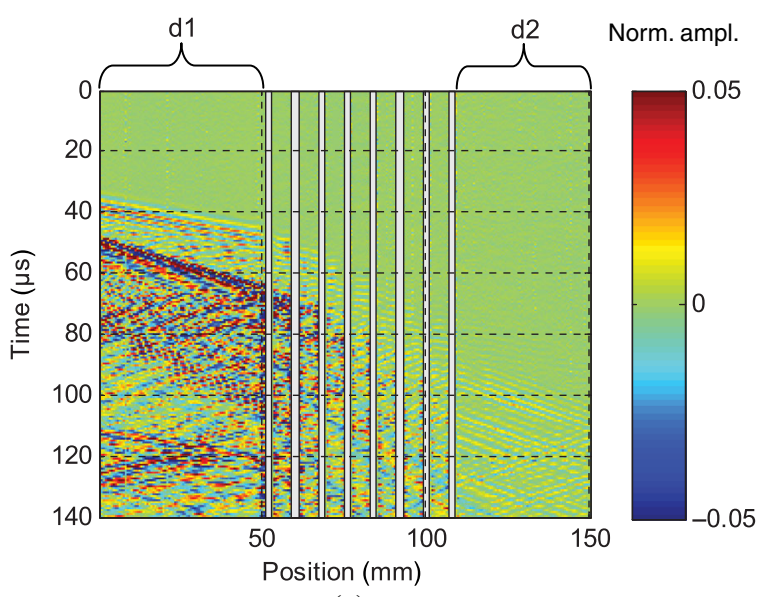

(a)
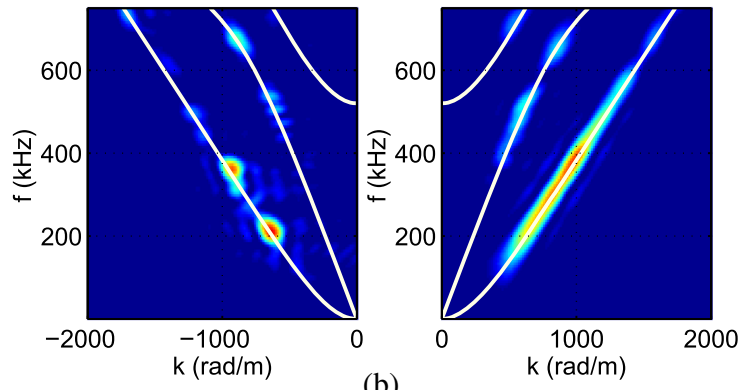

(b)

FIG. 3. (a) Out-of-plane displacement as a function of time $t$ and position $d$ along the dotted line in Fig. 1(a). White areas correspond to the crosslike cavities of the $\mathrm{PC} 1$ region. (b) Frequency $f$ vs wave number $k$ representation of the measured signals. Theoretical dispersion curves (in white) are superimposed. 
(details are provided in the Supplemental Material [39]). Results for the space-time evolution of the measured amplitudes are shown in Fig. 3(a). Because of the excitation, mainly antisymmetric $A 0 \mathrm{Lamb}$ waves are generated. However, Fig. 3(a) also clearly shows the presence of the $S 0$ mode (i.e., the faster waves visible in the $t=40 \mu \mathrm{s}$ region), derived from direct generation by the transducer. Strong reflections of the incident waves are clearly visible at a distance $d=50 \mathrm{~mm}$ (corresponding to the first cavity in the PC1 waveguide) due to Bragg scattering from PC1. Finally, Fig. 3(a) highlights a slight variation of the slope for the modes crossing the PC1 region, corresponding to a gradual decrease of the wave speed with the distance traveled in PC1.

The signals detected at various positions along the path are processed by applying a two-dimensional (2D) FFT and determining the energy values for each processed point. This enables us to obtain a frequency wave number representation [Fig. 3(b)]. Data are shown for the $d 1$ acquisition region of the plate (i.e., from $d=0$ up to the first cavity), with negative and positive values of the wave number $k_{x}$ corresponding to reflected and incident waves, respectively. This representation clearly identifies the energy distribution among the excited modes. The energy maxima of the reflected waves [Fig. 3(b), left panel] occur near the predicted BG frequency range, i.e. around 200 and $380 \mathrm{kHz}$, associated with the incident $A 0$ mode [Fig. 3(b), right panel]. The excited $S 0$-mode frequencies are greater than $450 \mathrm{kHz}$ [see Fig. 3(b)], i.e., outside the PC1 BG. In this frequency range, the polarization of the propagating waves in $\mathrm{PC} 1$ is predominantly in plane [see Fig. 2(b)]. Therefore, most of the $S 0$ wave field appears to be reflected since the SLDV setup is mainly sensitive to out-of-plane components (see also the Supplemental Material [39]). Numerically predicted dispersion curves (the white lines) are superimposed onto the experimental data, showing excellent agreement (see also the Supplemental Material [39]).

The $\mathrm{PC} 1$ region thus acts as a natural filter for frequencies in the $172-244 \mathrm{kHz}$ range. The excitation of a nonlinear elastic material with a monochromatic wave of frequency falling inside the $\mathrm{BG}$ of $\mathrm{PC} 1$ (e.g., $200 \mathrm{kHz}$ ) produces higher harmonics in the plate that can cross the PC1 barrier and enter the circular "chaotic cavity" [37]. Owing to its ergodic properties and negligible absorption, the latter is widely used in TR experiments to generate multiple reflections and a reverberant acoustic field, making a single transducer sufficient for signal acquisition $[37,42,43]$. The additional $C$-shaped phononic crystal structure (PC2) is designed to reflect the higher harmonics of the signal falling within the $\mathrm{BG}$ and to concentrate them in the geometric center of the mirror, thus enhancing their signal-to-noise ratio. Analysis of SLDV-measured signals filtered at the frequencies of interest shows that there is good energy concentration at the center of the mirror structure compared to peripheral regions in the chaotic cavity [39].

Additional FEM transmission simulations using the ABAQUS software are performed. The incoming wave is

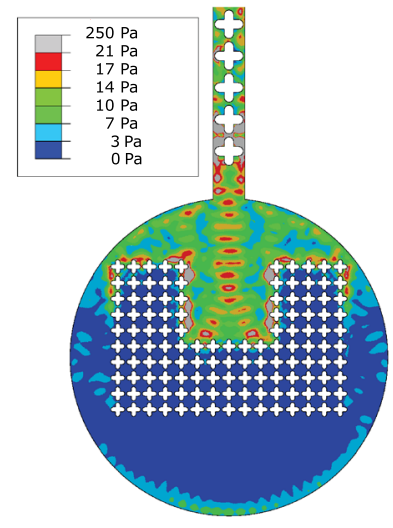

(a)

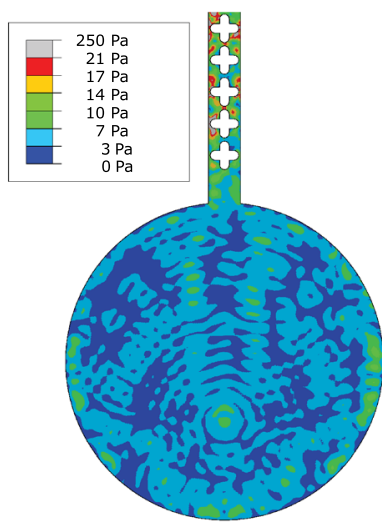

(b)
FIG. 4. Snapshots of the von Mises stress (in $\mathrm{Pa}$ ) inside the chaotic cavity showing the energy focusing by (a) the $C$-shaped phononic structure in comparison with (b) a homogeneous chaotic cavity at $t=160 \mu \mathrm{s}$.

the superposition of two quasimonocromatic waves centered around $f_{1}=200 \mathrm{kHz}$ and $f_{2}=400 \mathrm{kHz}$, respectively, with an imposed out-of-plane displacement of $1 \times 10^{-6} \mathrm{~mm}$ at point $E$. Two models are compared: one comprising both the filtering and focusing regions and another consisting of only the filtering region (i.e., with a homogeneous aluminum chaotic cavity). Figures 4(a) and 4(b) provide snapshots of the von Mises stress maps at $t=160 \mu \mathrm{s}$ for the two configurations. In the case of the chaotic cavity with the $C$-shaped structure, the formation of stationary waves occurs between the vertical portion of the mirror and the beginning of the waveguide. This allows the focusing of the energy of the wave at the frequency $f_{2}$ (i.e., the signature of the nonlinearity) to be enhanced.

The possibility of combining both the filtering and focusing functionalities of the device for TR-NEWS is now demonstrated. As discussed, the higher harmonics generated by the nonlinear source are transmitted through the $\mathrm{PC} 1$ region without the need for any postprocessing procedure (e.g., FFT). This allows the signal recorded by the sensor in the $C$-shaped mirror to be readily inverted and retransmitted into the sample. On the other hand, the PC2 region allows us to focus energy of the second harmonic in order to enhance the signal-to-noise ratio in TR.

To perform the TR experiment, two piezoelectric disk transducers (PZT 1, $1.25 \mathrm{~cm} / 1 \mathrm{MHz}$, and PZT 2, $5 \mathrm{~mm}$ ) are placed on the plate at points $C$ and $B$ [Fig. 1(a)], with the latter acting both as a receiver and as an actuator in the forward and backward TR propagation steps, respectively. Higher order harmonics are locally generated in the linear elastic plate by inducing the wave field to interact with an obstacle (e.g., a small cylinder, $8 \mathrm{~mm}$ in diameter and $20 \mathrm{~mm}$ high, placed on the surface of the sample at a point $D$ ) whose contact surface has been previously humidified. The emergence of nonlinearity can be ascribed to two different reasons: the first is the fact that the two surfaces are in "clapping" contact [44], mimicking the behavior of a 


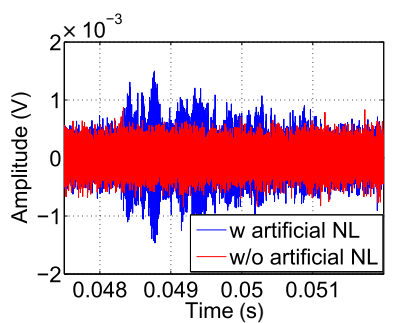

(a)

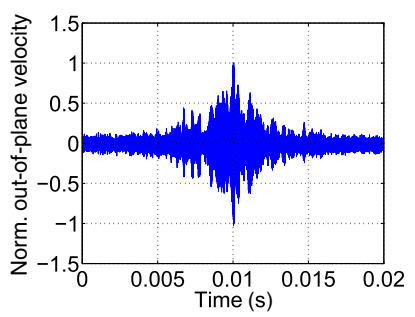

(c)

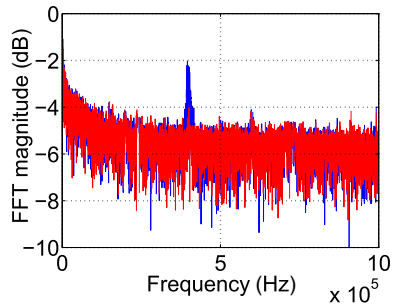

(b)

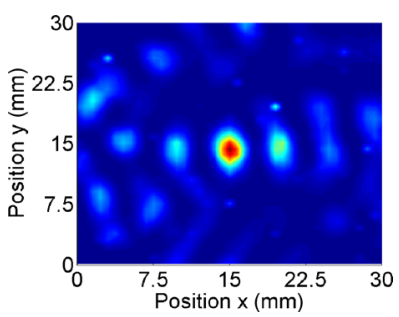

(d)
FIG. 5. (a) Time and (b) frequency domain representations of the signals without (red) and with (blue) the nonlinear (NL) source acquired inside the $C$-shaped mirror. Note the difference in frequency content. Evidence for (c) time and (d) spatial refocusing onto the nonlinear source location.

macroscopic crack in the sample; the second is due to the intrinsic nonlinear elastic behavior of water [45], amplified by the presence of the small cylinder. The sample (with and without the nonlinear element) is excited by a source of the form

$$
Y(t)=Y_{1}=A_{1} \sin \left(2 \pi f_{1} t\right) H\left(t_{0}\right),
$$

where $A_{1}$ is the amplitude of the sine function and $H\left(t_{0}\right)$ is the Hanning window centered in $t_{0}$ with a width corresponding to 21 cycles of the sine wave of the frequency $f_{1}$. The time and frequency domain representations of the signal received by PZT 2 are shown in Figs. 5(a) and 5(b), respectively. In the case without the nonlinear scatterer, only noise is recorded inside the cavity (the red signal), whereas, in the presence of the artificial nonlinearity, a resonance peak appears (the blue signal) around the second harmonic (i.e., $f_{2}=2 f_{1}=400 \mathrm{kHz}$ ).

The TR-NEWS experiment is thus performed as follows: the PZT 1 transducer emits a signal $Y=Y_{1}$ [Eq. (1)]; the signal detected by PZT 2 [the blue signal in Fig. 5(a)] is time reversed and transmitted back in the sample. SLDV measurements are performed on a spatial grid covering a $30 \times 30 \mathrm{~mm}^{2}$ region around the nonlinear source (removed in the back propagation experiment) consisting of $200 \times$ 200 equally spaced grid points. The laser vibrometer is positioned perpendicularly at $50 \mathrm{~cm}$ from the surface to record the out-of-plane velocities of the points over the target area. Multiple (128) measurements are performed and averaged for each node, to filter out part of the noise.

After the backward propagation, time compression of the signal and spatial focusing of the wave field are observed at the nonlinear scatterer location. Figure 5(c) reports an
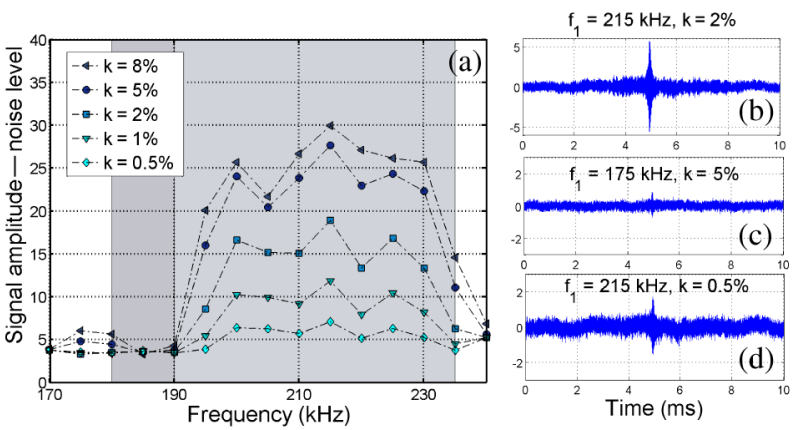

FIG. 6. Bandwidth of the proposed device for time reversal. (a) Quality of the focusing (signal-to-noise ratio) vs frequency. (b)-(d) Time-reversed signals for various nonlinearity levels and frequencies.

example of a time recompressed signal detected with the SLDV. Also, at the focal time, the spatial map of the recorded velocities reveals focusing at the location of the defect, with a considerable concentration of energy, as shown in Fig. 5(d).

The bandwidth and performance of the $C$-shaped mirror is evaluated by means of an additional experiment: PZT 1 is used to emit pulses [Eq. (1)] with variable frequencies in BG1 $\left(170<f_{1}<240 \mathrm{kHz}\right)$ and a controlled nonlinearity is introduced by replacing the nonlinear scatterer with a transducer PZT 3 emitting pulses [Eq. (1)] in the same time interval with amplitude $A_{2}=k A_{1}(k \ll 1)$ and frequency $f_{2}=2 f_{1}$, thus simulating the generation of second order harmonics. Signals received in the cavity are time reversed and the signal is detected by PZT 3. The quality of the focusing is evaluated as the ratio between the amplitude of the signal at the focal time and the root mean square (rms) of the signal excluding the peak. Results are shown in Fig. 6. Four frequency ranges are identified. (i) For $170<$ $f_{1}<180 \mathrm{kHz}$ and $235<f_{1}<240 \mathrm{kHz}$, the focusing is very poor even for high levels of nonlinearity (large $k$ ) since $f_{2}$ does not fall in BG2, so there is no effect of the mirror [see also Fig. 6(c)]. (ii) For $180<f_{1}<190$ kHz, focusing is poor, even though the mirror is expected to be effective. This is due to the presence of a band gap in $\mathrm{PC} 1$ at $f_{2}$ that prevents the second harmonic from reaching the device. (iii) For $190<f_{1}<235 \mathrm{kHz}$, the presence of the mirror $\left(f_{2}\right.$ falls in BG2) allows a significant improvement in the quality of the focusing [see also Figs. 6(b) and 6(d)]. Thus, the PC2 is effective even for very small nonlinearity levels ( $k=0.005)$, for which focusing is absent outside the mirror operating frequencies.

In conclusion, we have presented combined experimental and numerical results to demonstrate the feasibility of a novel passive sensor for signals generated by nonlinear elastic scatterers, such as cracks and delaminations. To do this, we have exploited the advanced frequency filtering and spatial focusing properties of phononic crystals, and we have proved the applicability of the sensor to time-reversal experiments that allow us to determine the spatial location 
of damage. A time-reversal experiment was carried out, showing good refocusing in time and space onto the nonlinear source, demonstrating the feasibility of the proposed device for damage localization in structures. The imaging results indicate that the PC mirror is necessary to achieve ultrasensitive detection, particularly when the nonlinear signature of the features to be localized is very small, and could provide additional functionalities such as frequency-selective focalization on multiple nonlinear scatterers with different characteristic frequencies.

In the future, we aim to improve the design of this PC sensor addressing issues such as optimized filter or focusing mirror designs, exploitation of multiple band gaps, or frequency tunability using piezoelectric patches, and its effective application to external tested structures with reduced signal losses. Nevertheless, the results presented in this Letter already provide the proof of concept for an efficient, portable damage sensor with applications for passive continuous structural health and acoustic emission monitoring in, e.g., civil engineering and the aerospace industry.

M. M. has received funding from the European Union's Horizon 2020 research and innovation program under Marie Skłodowska-Curie Grant Agreement No. 658483. A. K. has received funding from the European Union's Seventh Framework program for research and innovation under Marie Skłodowska-Curie Grant Agreement No. 609402-2020 researchers: Train to Move (T2M). N.M. P. is supported by European Research Council PoC 2015 "Silkene" No. 693670, by the European Commission H2020 under Graphene Flagship Core 1 No. 696656 (WP14 "Polymer Nanocomposites") and FET Proactive "Neurofibres" Grant No. 732344. F. B. is supported by Neurofibres Grant No. 732344 .

\section{*antonio.gliozzi@polito.it}

[1] B. Morvan, A. Tinel, A.-C. Hladky-Hennion, J. Vasseur, and B. Dubus, Appl. Phys. Lett. 96, 101905 (2010).

[2] M. S. Kushwaha, P. Halevi, L. Dobrzynski, and B. DjafariRouhani, Phys. Rev. Lett. 71, 2022 (1993).

[3] R. Martinez-Sala, J. Sancho, J. V. Sanchez, V. Gomez, J. Llinares, and F. Meseguer, Nature (London) 378, 241 (1995).

[4] S. Yang, J. H. Page, Z. Liu, M. L. Cowan, C. T. Chan, and P. Sheng, Phys. Rev. Lett. 93, 024301 (2004).

[5] M. Brun, S. Guenneau, A. B. Movchan, and D. Bigoni, J. Mech. Phys. Solids 58, 1212 (2010).

[6] A. S. Gliozzi, M. Miniaci, F. Bosia, N. M. Pugno, and M. Scalerandi, Appl. Phys. Lett. 107, 161902 (2015).

[7] S. Zhang, C. Xia, and N. Fang, Phys. Rev. Lett. 106, 024301 (2011).

[8] D. L. Sounas, R. Fleury, and A. Alù, Phys. Rev. Applied 4, 014005 (2015).

[9] W. Kan, V. M. García-Chocano, F. Cervera, B. Liang, X.-y. Zou, L.-1. Yin, J. Cheng, and J. Sánchez-Dehesa, Phys. Rev. Applied 3, 064019 (2015).
[10] S. Zhang, Y. Zhang, Y. Guo, Y. Leng, W. Feng, and W. Cao, Phys. Rev. Applied 5, 034006 (2016).

[11] X. Zhu, B. Liang, W. Kan, Y. Peng, and J. Cheng, Phys. Rev. Applied 5, 054015 (2016).

[12] P. Deymier, Acoustic Metamaterials and Phononic Crystals, Springer Series in Solid-State Sciences (Springer, Berlin, 2013).

[13] Y. Pennec, J. O. Vasseur, B. Djafari-Rouhani, L. Dobrzyński, and P. A. Deymier, Surf. Sci. Rep. 65, 229 (2010).

[14] M. Carrara, M. Cacan, J. Toussaint, M. Leamy, M. Ruzzene, and A. Erturk, Smart Mater. Struct. 22, 065004 (2013).

[15] K.-A. Van Den Abeele, P. A. Johnson, and A. Sutin, Research in Nondestructive Evaluation 12, 17 (2000).

[16] V. Y. Zaitsev, V. E. Gusev, V. Tournat, and P. Richard, Phys. Rev. Lett. 112, 108302 (2014).

[17] C. Payan, V. Garnier, and J. Moysan, J. Acoust. Soc. Am. 121, EL125 (2007).

[18] I. Solodov, J. Wackerl, K. P. Pfleiderer, and G. Busse, Appl. Phys. Lett. 84, 5386 (2004).

[19] Y. Ohara, T. Mihara, R. Sasaki, T. Ogata, S. Yamamoto, Y. Kishimoto, and K. Yamanaka, Appl. Phys. Lett. 90, 011902 (2007).

[20] G. Renaud, S. Callé, and M. Defontaine, Appl. Phys. Lett. 94, 011905 (2009).

[21] P. Finkel, A. G. Zhou, S. Basu, O. Yeheskel, and M. W. Barsoum, Appl. Phys. Lett. 94, 241904 (2009).

[22] C. Trarieux, S. Callé, H. Moreschi, G. Renaud, and M. Defontaine, Appl. Phys. Lett. 105, 264103 (2014).

[23] M. Scalerandi, A. S. Gliozzi, M. Ait Ouarabi, and F. Boubenider, Appl. Phys. Lett. 108, 214103 (2016).

[24] J. Chen, J. Kim, K. Kurtis, and L. Jacobs, J. Acoust. Soc. Am. 130, 2728 (2011).

[25] M. Scalerandi, A. Gliozzi, C. L. E. Bruno, D. Masera, and P. Bocca, Appl. Phys. Lett. 92, 101912 (2008).

[26] M. Scalerandi, M. Griffa, P. Antonaci, M. Wyrzykowski, and P. Lura, J. Appl. Phys. 113, 154902 (2013).

[27] M. Ait Ouarabi, F. Boubenider, A. S. Gliozzi, and M. Scalerandi, Phys. Rev. B 94, 134103 (2016).

[28] N. Krohn, K. Pfleiderer, R. Stoessel, I. Solodov, and G. Busse, Acoust. Imaging 27, 91 (2004).

[29] Z. Ficek and P. D. Drummond, Phys. Today 50, No. 9, 34 (1997).

[30] T. J. Ulrich, P. A. Johnson, and A. M. Sutin, J. Acoust. Soc. Am. 119, 1514 (2006).

[31] A. S. Gliozzi, M. Griffa, and M. Scalerandi, J. Acoust. Soc. Am. 120, 2506 (2006).

[32] T. J. Ulrich, A. Sutin, R. Guyer, and P. Johnson, Int. J. Nonlinear Mech. 43, 209 (2008).

[33] C. Prada, E. Kerbrat, D. Cassereau, and M. Fink, Inverse Probl. 18, 1761 (2002).

[34] T. Goursolle, S. D. Santos, O. B. Matar, and S. Callé, Int. J. Nonlinear Mech. 43, 170 (2008).

[35] T. J Ulrich, P. A. Johnson, and R. A. Guyer, Phys. Rev. Lett. 98, 104301 (2007).

[36] G. Zumpano and M. Meo, Int. J. Solids Struct. 44, 3666 (2007).

[37] O. Bou Matar, Y. F. Li, and K. Van Den Abeele, Appl. Phys. Lett. 95, 141913 (2009).

[38] M. Miniaci, A. Marzani, N. Testoni, and L. De Marchi, Ultrasonics 56, 251 (2015). 
[39] See Supplemental Material at http://link.aps.org/ supplemental/10.1103/PhysRevLett.118.214301, which includes Ref. [40], for further details on the physical mechanisms and functionalities of the phononic crystal elements described in the text.

[40] A. Krushynska, V. Kouznetsova, and M. Geers, J. Mech. Phys. Solids 71, 179 (2014).

[41] M. Collet, M. Ouisse, M. Ruzzene, and M. Ichchou, Int. J. Solids Struct. 48, 2837 (2011).
[42] C. Draeger and M. Fink, Phys. Rev. Lett. 79, 407 (1997).

[43] A. M. Sutin, J. A. TenCate, and P. A. Johnson, J. Acoust. Soc. Am. 116, 2779 (2004).

[44] I. Solodov, D. Döring, and G. Busse, Stroj. Vestn - J. Mech. E. 57, 169 (2011).

[45] C. Pecorari and M. Poznic, Proc. R. Soc. A 462, 769 (2006). 


\title{
Supplemental Material for "Proof of concept for an ultrasensitive technique to detect and localize sources of nonlinear elasticity using phononic crystals"
}

\author{
M. Miniaci, ${ }^{1}$ A. S. Gliozzi, ${ }^{2,}{ }^{*}$ B. Morvan, ${ }^{1}$ A. Krushynska,${ }^{3}$ \\ F. Bosia, ${ }^{3}$ M. Scalerandi, ${ }^{2}$ and N. M. Pugno ${ }^{4,5,6}$ \\ ${ }^{1}$ University of Le Havre, Laboratoire Ondes et Milieux Complexes, \\ UMR CNRS 6294, 75 Rue Bellot, 76600 Le Havre, France \\ ${ }^{2}$ Department of Applied Science and Technology, Politecnico di Torino, \\ Corso Duca degli Abruzzi 24, 10129 Torino, Italy \\ ${ }^{3}$ Department of Physics, University of Torino, \\ Via Pietro Giuria 1, 10125 Torino, Italy \\ ${ }^{4}$ Laboratory of Bio-Inspired and Graphene Nanomechanics, \\ Department of Civil, Environmental and Mechanical Engineering, \\ University of Trento, Via Mesiano 77, 38123 Trento, Italy \\ ${ }^{5}$ School of Engineering and Materials Science, \\ Queen Mary University of London, \\ Mile End Road, London E1 4NS, United Kingdom \\ ${ }^{6}$ Ket Lab, Edoardo Amaldi Foudation, Italian Space Agency, \\ Via del Politecnico snc, 00133 Rome, Italy
}

(Dated: May 12, 2017)

*Electronic address: antonio.gliozzi@polito.it 


\section{SAMPLES AND EXPERIMENTAL CONFIGURATION}

A schematic representation of the experimental specimen is shown in Fig. SM1, along with its dimensions. The specimen can be formally divided into three parts: the first represents the sample under investigation and consists of an aluminium plate $(\rho=2700$ $\mathrm{kg} / \mathrm{m}^{3}, E=70 \mathrm{GPa}$ and $\nu=0.33$ ) while the other two remaining portions (regions 1 and 2 in the figure) represent the device, consisting of two phononic crystals regions (referred in the following as $\mathrm{PC} 1$ and $\mathrm{PC} 2$, respectively). These two regions are composed of 1D and 2D-periodic arrangements of cross-like holes fabricated through waterjet cutting, with different lattice parameters, depending on the filtering (PC1) or reflecting (PC2) function they are designed for. The lattice parameters are specified in the main text.

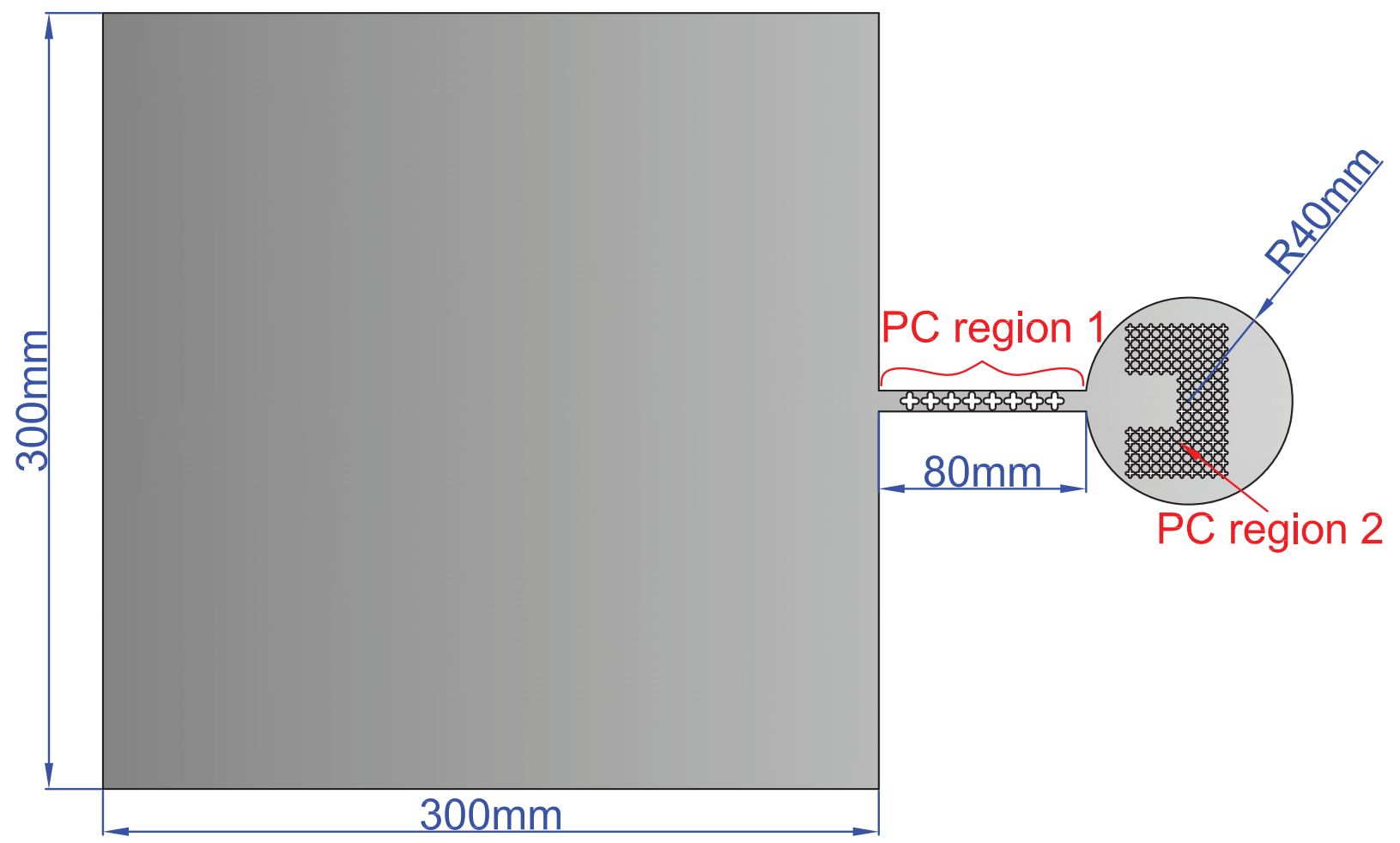

FIG. SM1: Schematic representation of the specimen composed of an aluminium plate connected to a filtering phononic crystal region (PC1) and a chaotic cavity with an additional focusing phononic crystal-based structure (PC2). Cross-like hole dimensions for $\mathrm{PC} 1$ and $\mathrm{PC} 2$ are given in the main text.

The sample and device thicknesses are the same and all are obtained via water-jet cutting starting from a unique pristine and undamaged aluminium plate, exhibiting linear elastic 
properties.

\section{NUMERICAL RESULTS}

Dispersion diagrams are computed numerically for the considered phononic crystal structures. The application of the Bloch-Floquet theory allows to consider only one so-called unit cell, i.e. the PC representative geometry for computing the dispersion diagrams [1]. Full 3D models are implemented to capture all the possible wave modes. Cells are meshed by means of 4-node tetrahedral elements of maximum size $L_{F E}=0.5 \mathrm{~mm}$ in order to provide accurate eigensolutions up to the maximum frequency of interest.

The band structures are derived assuming periodic (in the $x$-direction) and free (in the $y$-direction) boundary conditions at the edges of the cell domains for case PC1 and periodic (both in $x$ - and $y$-directions) boundary conditions for PC2. In both cases free boundary conditions are imposed at the top and bottom surfaces of the cells. The resulting eigenvalue problem $\left(\mathbf{K}-\omega^{2} \mathbf{M}\right) \mathbf{u}=\mathbf{0}[2]$ is solved by varying the wave vector $\vec{k}=\left\{k_{x}, k_{y}\right\}$ values along the first irreducible Brillouin zone boundary $\Gamma-X$ for PC1 (since the periodicity is only in the $x$-direction) and the path $\Gamma-X-M$ for $\mathrm{PC} 2$ (since the periodicity is two-dimensional in this case).

Figures SM2a and SM2b present the band structures for the unit cells PC1 and PC2 in terms of reduced wavevector $k^{*}=\left[k_{x} \cdot a_{c} / \pi ; k_{y} \cdot a_{c} / \pi\right]$ with $a_{c}$ denoting $A$ for PC1 and $a$ for PC2 (lattice parameters are given in the main text). For the PC1 structure, four BGs (grey rectangles) exist in the $[0-500] \mathrm{kHz}$ frequency range, with the larger one extending from $172 \mathrm{kHz}$ to $244 \mathrm{kHz}$ (Fig. SM2a). The other three smaller BGs have central frequencies at around 150, $285 \mathrm{kHz}$ and $380 \mathrm{kHz}$, in excellent agreement with the experimentally measured ones (see main text). Fig. SM2b shows the dispersion diagram for the PC2 structure, where a single $\mathrm{BG}$ is observed in the range $360<f<470 \mathrm{kHz}$.

In order to highlight the in-plane or out-of-plane polarization of the modes in the dispersion diagram for $\mathrm{PC} 1$, we define a polarization factor:

$$
p=\frac{\int_{V}\left(\left|u_{z}\right|\right)^{2} d V}{\int_{V}\left(\left|u_{x}\right|^{2}+\left|u_{y}\right|^{2}+\left|u_{z}\right|^{2}\right) d V},
$$

where $V$ is the volume of the unit cell, $u_{x}, u_{y}$ and $u_{z}$ are the displacement components along $x, y$ and $z$ axes, respectively. The dispersion curves in Fig. SM3 are shaded accordingly, 


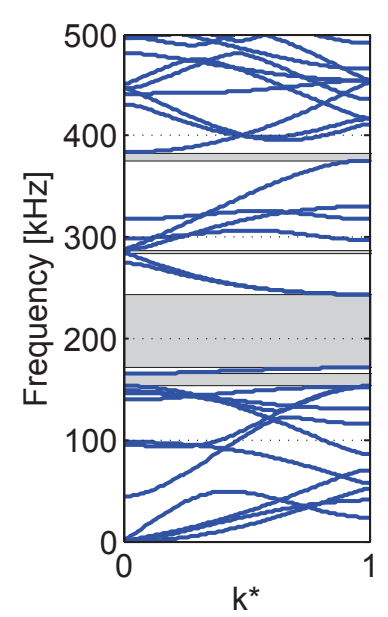

(a)

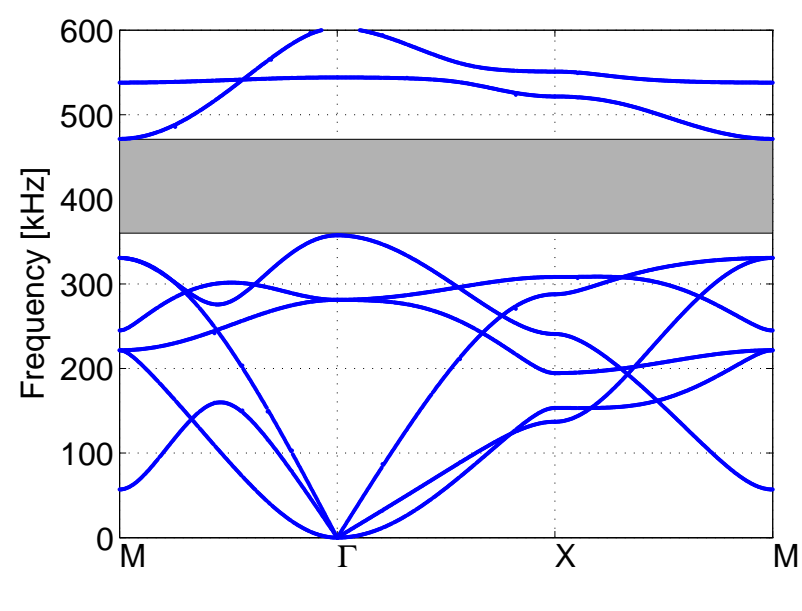

(b)

FIG. SM2: (a) Numerically-predicted dispersion band structure for the PC1 region. (b) Numerically-predicted band structure for $\mathrm{PC} 2$ region.

with colours varying from 0 (blue) to 1 (red), making the colour change from blue to red gradually. Thus colours close to red indicate vibration modes that are dominantly polarized out-of-plane, while the colours close to blue are predominantly polarized in-plane. This allows us to infer that although a mode is present between 325 and $375 \mathrm{kHz}$, its behaviour is mainly dominated by out-of-plane deformation. This implies that in-plane modes are strongly attenuated in these frequency regions since there are no modes allowing the energy propagation via in-plane deformations. On the contrary at frequencies larger than $450 \mathrm{kHz}$ energy propagation in the device occurs prevalently via in-plane deformations.

\section{FREQUENCY FILTERING EXPERIMENTS}

In this section, we describe in further detail the experimental setups for the study of the dynamic properties of the phononic crystal waveguide.

The transmission spectrum of the PC1 region is first investigated by means of a pitchcatch experiment [3] according to the schematic representation of Fig. SM4a. A broadband longitudinal piezocomposite transducer (Krautkramer transducer with a $1.25 \mathrm{~cm}$ diameter and $1 \mathrm{MHz}$ central frequency) is selected as a source and placed at point $\mathrm{E}$ on the plate. The transducer is capable of introducing the desired excitations at the frequencies/wavelengths of interest for this study. Two $5 \mathrm{~mm}$ diameter PZT disks with wrap-around contacts from 


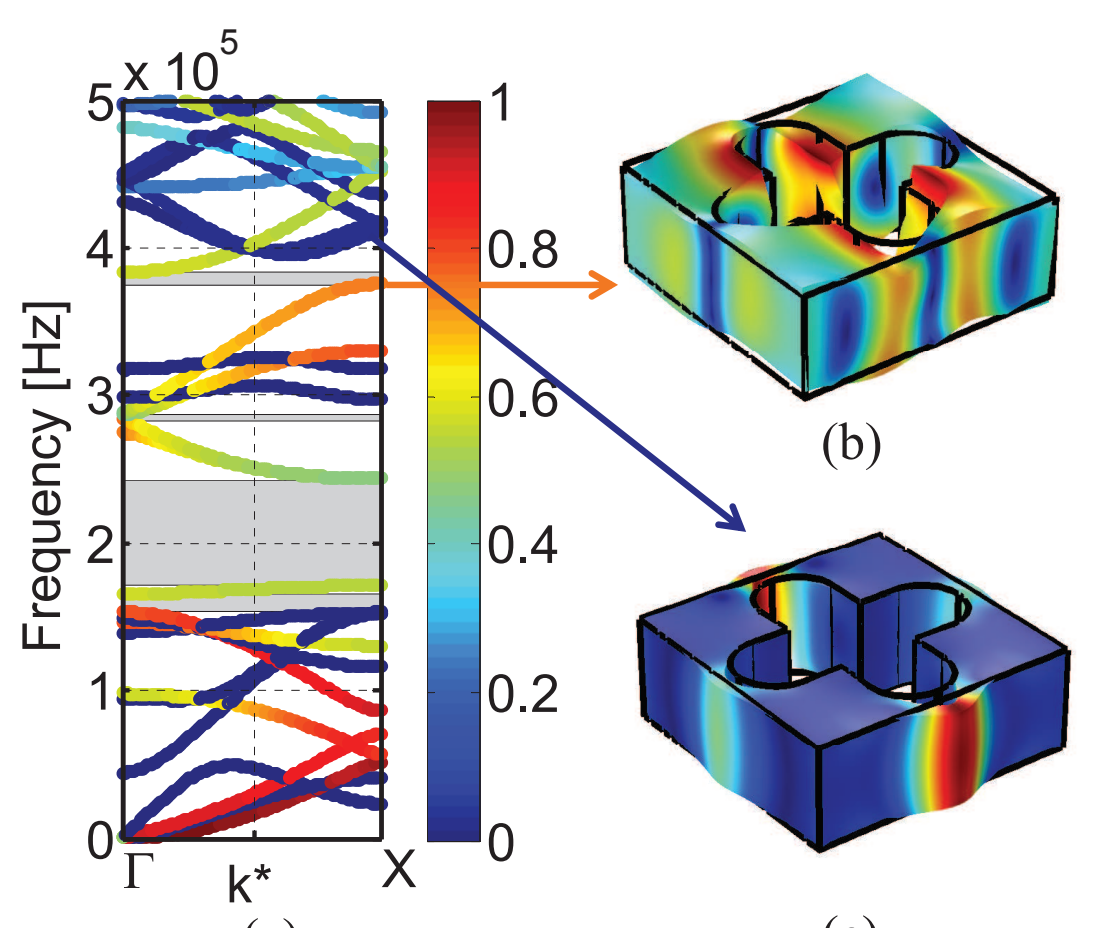

(a)

(c)

FIG. SM3: (a) Dispersion diagram showing in-plane and out of plane polarized modes for the PC1 unit cell. The colours close to red mean that the vibration modes are dominantly out-of plane, while colours close to blue are dominantly in-plane polarized. b) and c) show two corresponding mode shapes.

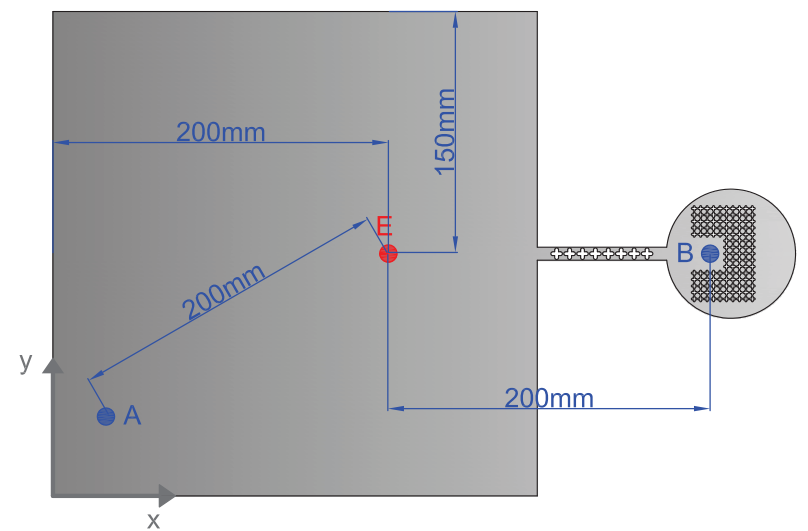

(a)

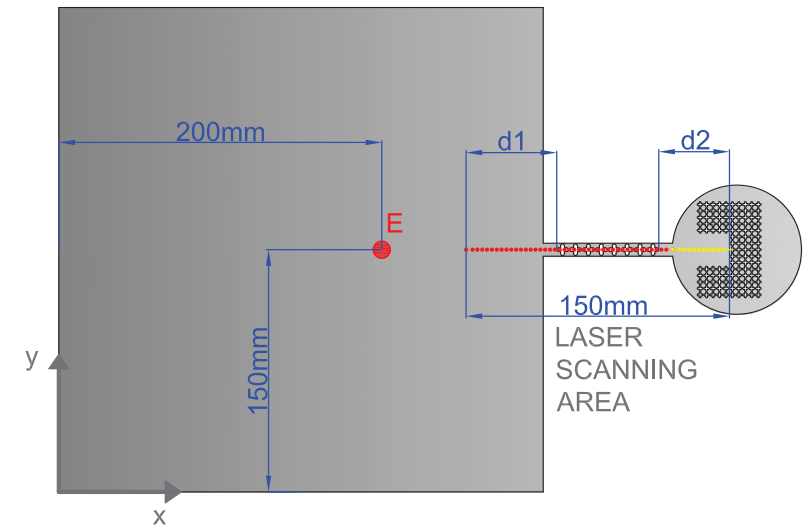

(b)

FIG. SM4: Experimental setup for (a) the pitch-catch configuration and (b) the Scanning Laser Doppler Vibrometer (SLDM) acquisition line. 
$\mathrm{PI}^{\circledR}[4]$ are chosen as sensors and placed at points $\mathrm{A}$ and $\mathrm{B}$ on the plate. Lamb waves are excited at point $\mathrm{E}(E \equiv[200,150] \mathrm{mm})$ by injecting energy via a broadband frequency excitation, with a flat spectrum between $50 \mathrm{kHz}$ and $450 \mathrm{kHz}$. The desired source function is the sum of four Hanning modulated sine functions (3-cycles) centered at frequencies $0.5 \cdot f_{0}$, $1 \cdot f_{0}, 1.5 \cdot f_{0}$ and $2 \cdot f_{0}$, where $f_{0}=200 \mathrm{kHz}$. Signals are generated using an Agilent arbitrary waveform generator 33220A. Corresponding signals are detected inside the plate at point A and after the PC1 region at point B (Fig. SM4a), both $200 \mathrm{~mm}$ from the emitting transducer E. The propagation time is set to $200 \mu \mathrm{s}$ in order to allow wave reflections from all the plate edges. The wavefield is constrained to pass through the PC1 zone to reach B from E. Thus, the spectral content of the signal recorded in B reflects the filtering action of the PC1 region (see Fig. 2a of the main text for the results).

To further investigate the dynamical properties of the PC1 region, a second experiment is performed with the set-up configuration shown in Fig. SM4b. A pulse generator (Sofranel 5077PR) delivers a very short pulse voltage (about $300 \mathrm{~V}$ for $50 \mathrm{~ns}$ ) to the Krautkramer transducer positioned at E. A Scanning Laser Doppler Vibrometer (SLDV - Polytech model OFV 5000) is used to measure the normal component of the velocity at the surface of the plate. A $1 \mathrm{D}$ scan along the $x$-direction (the dotted path highlighted in Fig. SM4b) is performed with a spatial step of $0.2 \mathrm{~mm}$. At each position along the scanning line a signal is recorded, averaging 256 times to improve the signal-to-noise ratio. The sensitivity of the SLDV is set to $50 \mathrm{~mm} / \mathrm{s} / \mathrm{V}$. Propagation time is again set to $200 \mu \mathrm{s}$ in order to allow wave reflections from all the plate edges, resulting in multiple waves impinging both the PC1 and PC2 regions. The total number of points recorded by the laser are in this case 750, spaced by $\Delta x=0.2 \mathrm{~mm}$. The total length scanned by the laser is $L=15 \mathrm{~cm}$, starting $5 \mathrm{~cm}$ away from point $E$ in the plate and ending at the left boundary of the mirror (Fig. SM4b).

Data are processed for two regions of the plate, i.e along the paths $d 1$ and $d 2$ in Fig. SM4b, in order to analyse the spectral content of waves incident on and reflected by the two phononic crystal regions PC1 and PC2, respectively.

In particular, the signals referring to the $d 1$ scan points are time-windowed as follows: $t 1=$ $[0: 65] \mu$ s to examine the frequency content of the incident waves to PC1 and $t 2=[0: 90]$ $\mu \mathrm{s}$ to study the frequency content of the reflected by PC1 waves. This temporal windowing is used to exclude, as far as possible, the portion of the signal corresponding to multiple reflections from the edges of the plate/cross-like holes (see the spatio-temporal representation 


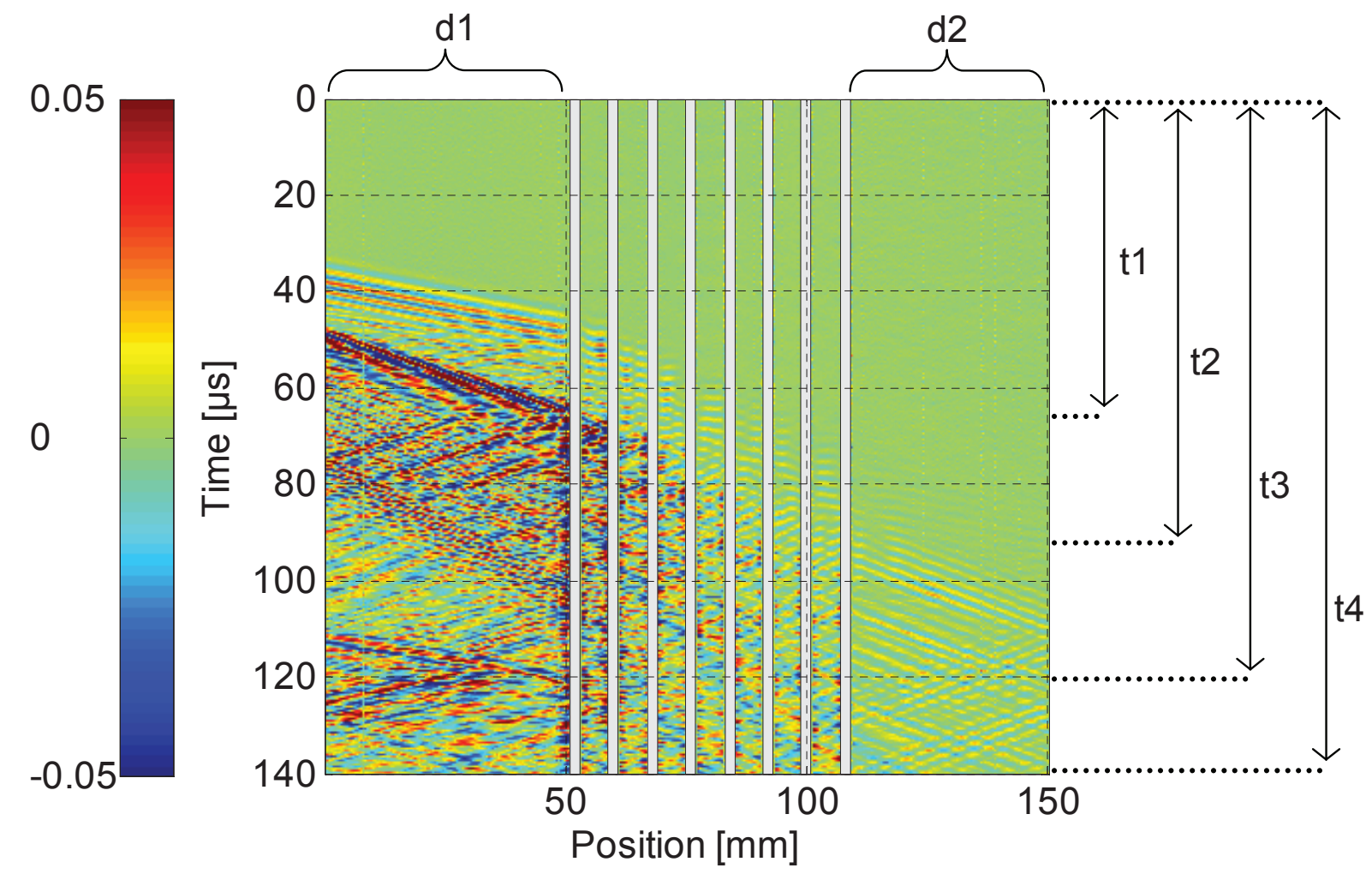

FIG. SM5: Space-time evolution of the out-of-plane displacement recorded by point-by-point Scanning Laser Doppler Vibrometer acquisitions along the dotted path highlighted in Fig. SM4b. White boxes correspond to the cross-like holes of the first PC region in the waveguide. Spatial $(\mathrm{d} 1, \mathrm{~d} 2)$ and temporal $(\mathrm{t} 1, \mathrm{t} 2, \mathrm{t} 3, \mathrm{t} 4)$ parameters for the signal processing of Fig. SM6 are reported

in Fig. SM5 corresponding to signals acquired in $d 1$ for times $t 1$ and $t 2$, respectively). Then, 2D Fourier transforms are applied on the windowed signals. The resulting frequency vs. wavenumber representations are shown in Fig. SM6a, in which negative (left panel) and positive (right panel) values of the wavenumber $k_{x}$ provide information about reflected and incident waves in $\mathrm{PC} 1$, respectively.

The same procedure is applied to signals registered at points belonging to $d 2$ with the following time windowing: $t 3=[0: 120] \mu$ s for examining the frequency content of the incident waves to PC2 and $t 4=[0: 140] \mu$ s to study the frequency content of the reflected waves by PC2. Similarly, the resulting wavenumber-frequency representations are shown in Fig. SM6b. 

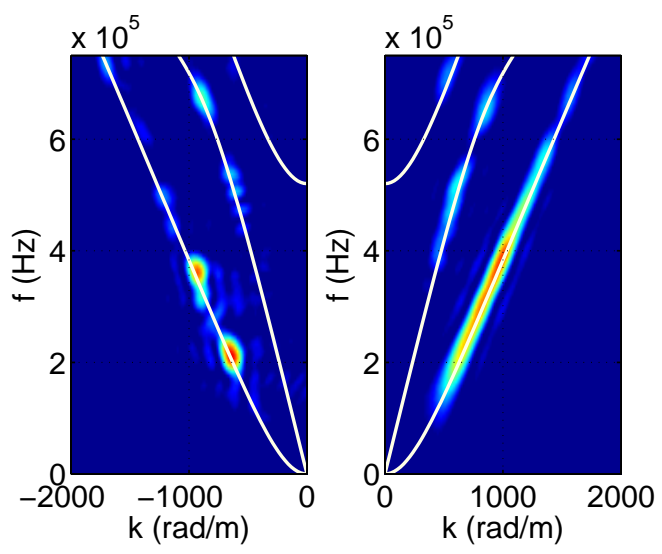

(a)
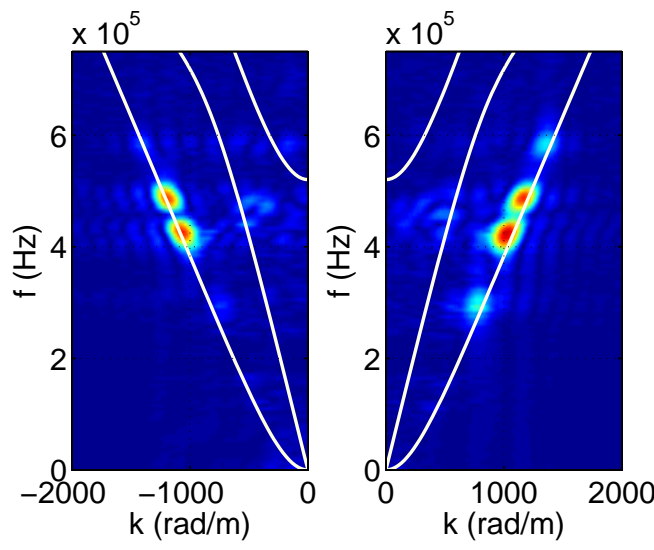

(b)

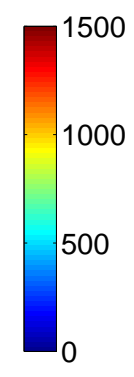

(c)

FIG. SM6: Wavenumber-frequency representation of the measured spatio-temporal signals for (a) $d 1$ line scan and (b) $d 2$ line scan presented in Fig. SM5. (c) The color map represents the amplitudes (arbitrary units); the theoretical dispersion curves are also presented for comparison (white lines).

\section{ENERGY FOCUSING EXPERIMENTS}

The efficiency of the PC2 region in focusing incoming wave energy is now investigated. The C-shaped structure is designed to reflect the spectral part of the signal with frequencies within a phononic crystal BG and to concentrate it in the geometric centre of the mirror. The idea is to take advantage of this element to enhance the spectral content of a selected frequency band in the spectrum of a recorded signal and to study the part of the signal generated by the nonlinearities present in the sample.

Figure SM6 shows the frequency-wavenumber $(f-k)$ representation of signals measured along the central axis of the mirror before, within and beyond the PC1 region (see Fig. SM4b) and clearly illustrates the operating BG frequencies of the PC1 and of the C-shaped $\mathrm{PC} 2$ regions. Although the incoming energy (positive values of $k$ ) contains multiple frequencies (spanning from 100 to $600 \mathrm{kHz}$ - see right panel of Fig. SM6a), the energy reflected by the barrier basically corresponds to those of the BGs.

The energy distribution in the area surrounded by the PC2 structure is also investigated to determine the existence of a spatial point which plays the role of focus of the mirror, i.e. where reflected energy is concentrated. Each signal $u_{i}(t)$ recorded in one of the laser positions $(i=1, \ldots, 750)$ is analysed at successive time intervals starting from $t_{j}(j=1, \ldots, 50$, each 

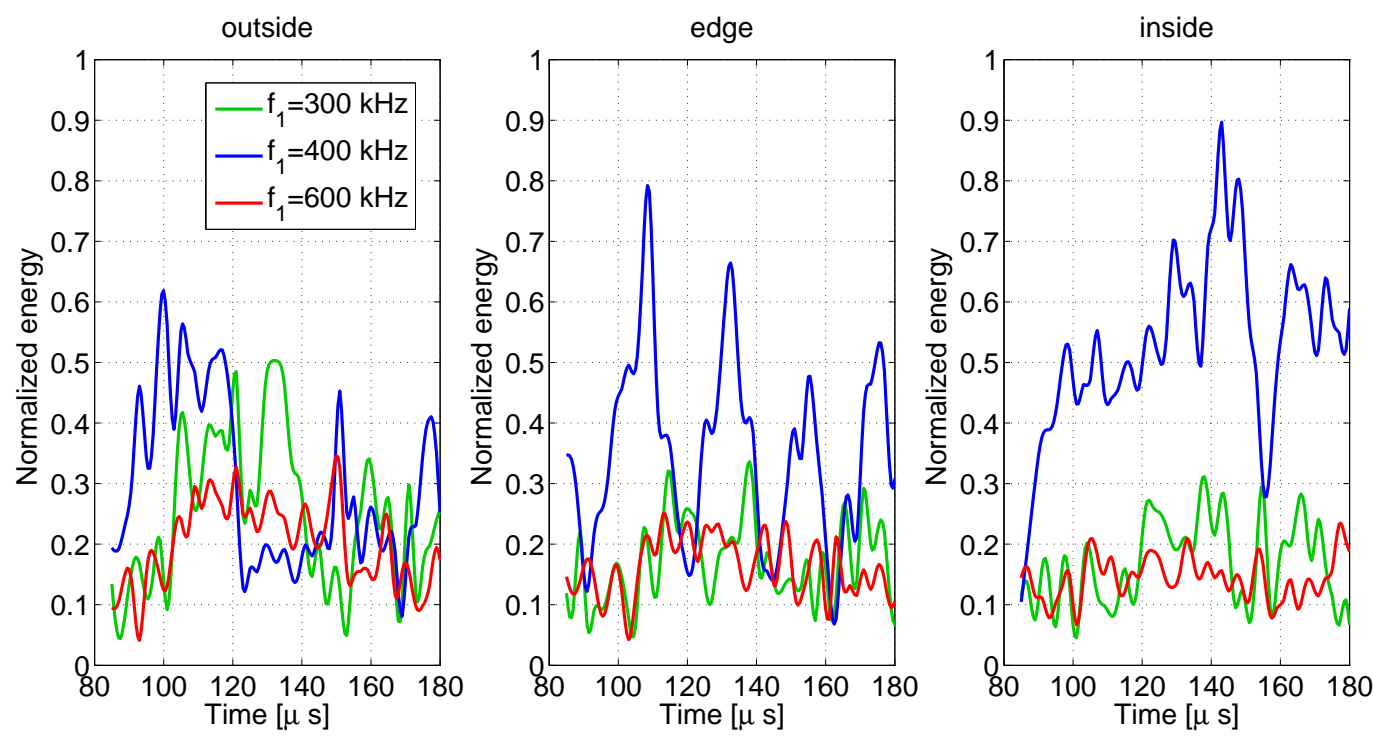

FIG. SM7: Spectral energy content as function of time at three selected frequencies $\left(f_{1}, f_{2}\right.$ and $f_{3}$ ) for signals recorded in three different points in the $\mathrm{PC} 2$ region: (a) outside, (b) at the edge and (c) inside the C-shaped cavity. It is apparent that inside the C-shaped mirror the $400 \mathrm{kHz}$ component, corresponding to the PC2 BG, is strongly enhanced.

separated by $2 \mu \mathrm{s})$ and of duration $\Delta t=10 \mu \mathrm{s}(j=1, \ldots, 50)$. We start from the first arrival of the wave front (approximately at $t_{i n i}=80 \mu \mathrm{s}$ ) and end at $t_{\text {fin }}=200 \mu \mathrm{s}$. Each portion of the $i^{t h}$ signal $u_{i}\left(t_{j}+\Delta t\right)$ is filtered at three representative frequencies $f_{1}=300$ $\mathrm{kHz}$ (below the BG of PC2), $f_{2}=400 \mathrm{kHz}$ (within the BG of PC2) and $f_{3}=500 \mathrm{kHz}$ (above the $\mathrm{BG}$ of $\mathrm{PC} 2$ ) and their weight is calculated, normalising it to the total spectral energy of the incident wave. Interestingly, the result depends on the position where the signal is recorded (i.e. on the $i$ index). Fig. SM7 shows the spectral content as a function of time for three different points: the first (Fig. SM7a) is chosen in the chaotic cavity but outside the C-shaped mirror, the second (Fig. SM7b) is at the edge of the C-shaped mirror, and the third (Fig. SM7c) is in the region enclosed by the mirror. After its first arrival, the signal filtered around $f_{2}=400 \mathrm{kHz}$ becomes dominant with respect to the other contributions in the region surrounded by the mirror (Fig. SM7b-c) due to enhanced reflection by the PC2 mirror. In the second subplot a periodic structure is visible (a similar behaviour is observed at other points, not reported here for brevity), confirming that a stationary wave or pulsing focal points are forming. 


\section{NUMERICAL TIME TRANSIENT SIMULATIONS}

To further investigate the spatial energy distribution inside the mirror structure, FEM transmission simulations by means of the commercial software ABAQUS are performed. The incoming wave is described by an injection function given by superposition of two quasi-monocromatic waves centred at $f_{1}=200 \mathrm{kHz}$ and $f_{2}=400 \mathrm{kHz}$, respectively. The two frequencies correspond to the fundamental and second harmonic, respectively. The resulting function has the following form:

$$
Y(t)=Y_{1}+Y_{2}=A_{1} \sin \left(2 \pi f_{1} t\right) * H\left(t_{0}\right)+A_{2} \sin \left(2 \pi f_{2} t\right) * H\left(t_{0}\right)
$$

where $A_{i}$ are the amplitudes of the sine functions and $H\left(t_{0}\right)$ are Hanning window functions centered in $t_{0}$, and set to a width corresponding to 21 cycles of the sine wave of the fundamental frequency $f_{1}$. Two FEM models are compared: a model comprising both the filtering and the focusing regions and another consisting of only the PC1 region (i.e. with a homogeneous aluminium chaotic cavity). To ensure accuracy up to the maximum frequency of interest, a mesh with approximately 5 million linear hexahedral elements of type C3D8R is used. Geometric and mechanical parameters are the same as described in the previous section for real experiments. PZT excitation is simulated by means of an imposed displacement of $1 \times 10^{-6} \mathrm{~mm}$ in the out of plane $z$-direction. Lamb waves are excited at the 1.25 $\mathrm{cm} / 1 \mathrm{MHz}$ Kautkramer transducer location. The time history and frequency content of the exciting signal are chosen in accordance with the previous experiment and consist of a sum of Hanning modulated 21 sine cycles centred at 200 and $400 \mathrm{kHz}$. This pulse is chosen to highlight the filtering/reflecting capabilities of the PC1 and PC2 regions, respectively. Time transient explicit simulations (0.1 ms in duration) are performed in order to allow multiple Lamb wave reflections to take place. Results are discussed in the main text.

\section{SOURCE LOCALIZATION VIA NONLINEAR TIME REVERSAL}

In the main paper, the possibility of combining the two mentioned functionalities of the phononic crystal elements for nonlinear NDT purposes, in particular for NEWS-TR, is demonstrated (as shown in Fig.5 of the main paper). Here the robustness of the TR procedure is verified by investigating the range of amplitudes of the injected and generated 
nonlinear signals (i.e. their ratio) for which focusing on the nonlinear scatterer position occurs. In order to better control the amplitude of the nonlinear source, it is replaced by an extra piezoelectric transducer at the same position, as also done for the analysis of the efficency of the mirror (Fig.6 of the main paper).

A $1.25 \mathrm{~cm} / 1 \mathrm{MHz}$ Krautkramer transducer (PZT 1) is used to inject pulses

$$
Y(t)=Y_{1}=A_{1} \sin \left(2 \pi f_{1} t\right) * H\left(t_{0}\right)
$$

where $A_{1}$ is the amplitude of the sine function and $H\left(t_{0}\right)$ is the Hanning window centered in $t_{0}$ and set to a width corresponding to 21 cycles of the sine wave of the fundamental frequency, $f_{1}=200 \mathrm{kHz}$ in BG1. A controlled nonlinearity is introduced by replacing the nonlinear scatterer with an identical transducer (PZT 3) emitting pulses in the same time interval with varying amplitudes $A_{2}=k A_{1}(k<<1)$ and frequency $f_{2}=2 f_{1}$, thus simulating the generation of second order harmonics. A $5 \mathrm{~mm}$ disk piezoelectric transducer (PZT 2) is placed on the plate at the center of the C-shaped mirror on the surface of the cavity as shown in Fig. SM8. PZT 2 acts both as a receiver and as an actuator in the forward and backward TR propagation steps, respectively. Signals received in the cavity are time reversed and the signal is detected by PZT 3. The quality of the focusing is evaluated as the ratio between the amplitude of the signal at the focal time and the Root Mean Square (RMS) of the signal. Results are shown in Fig.SM9.

Excellent temporal re-compression of the signal at the nonlinear location can be observed for $A_{2} / A_{1}$ ratios down to $3 \%$. The presence of the mirror still allows focusing down to a nonlinearity ratio $k=A_{2} / A_{1} \approx 0.005$. Weaker NL scatterers are not identifiable. For strongly nonlinear scatterers larger than $3 \%$ the focusing indicator is constant. The results presented here are in agreement with the findings reported in Fig. 6 of the main text and the slight differences are ascribable in the positioning of PZT 1 and PZT 2.

\section{References}

[1] M. Collet, M. Ouisse, M. Ruzzene, and M. Ichchou, International Journal of Solids and Structures 48, 2837 (2011). 


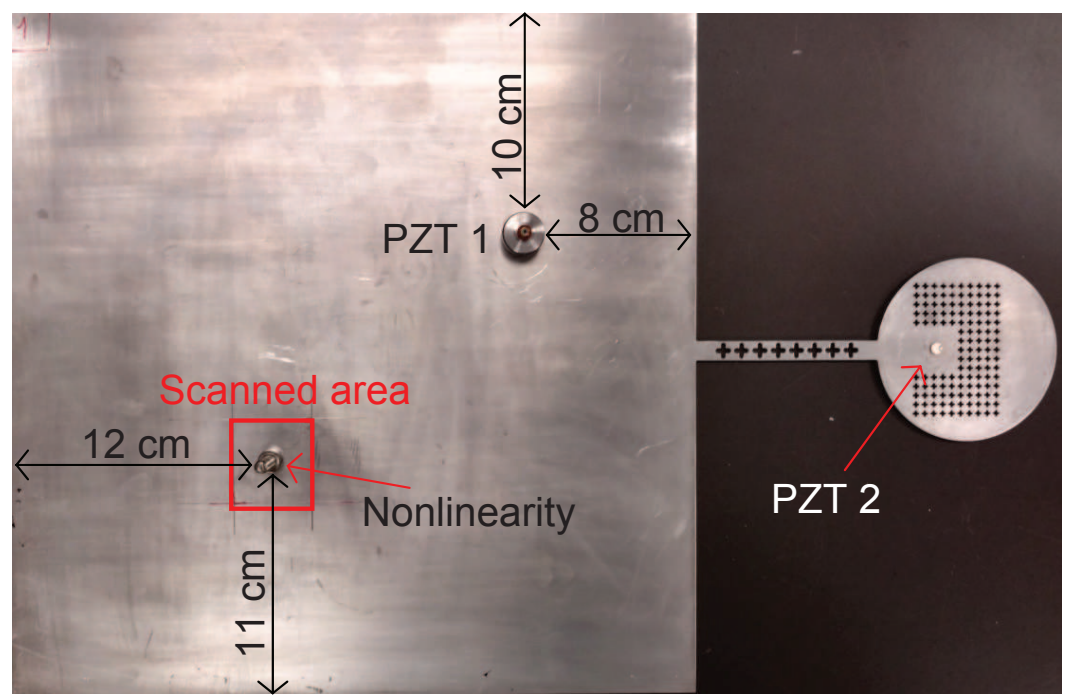

FIG. SM8: Configuration for the TR experiment in which a nonlinear source is simulated by means of a transducer PZT 3 (simulating the nonlinearity).

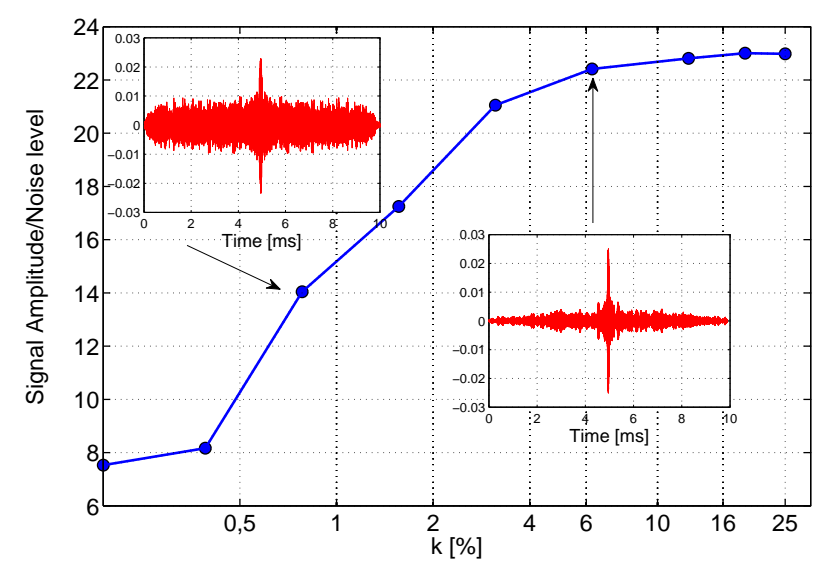

FIG. SM9: Ratio between the amplitudes of the focal point and of the signal RMS of the time reversed signals as a function of the ratio between the amplitudes of the second harmonic A2 and of the fundamental frequency A1.

[2] A. Krushynska, V. Kouznetsova, and M. Geers, Journal of the Mechanics and Physics of Solids 71, 179 (2014).

[3] M. Miniaci, A. Marzani, N. Testoni, and L. De Marchi, Ultrasonics 56, 251 (2015).

[4] www.piceramic.com 